\title{
Increase the Lifetime of Wireless Sensor Network using Clustering and Compression
}

\author{
Satyajeet R. Shinge \\ Department of Computer Engg. \\ Pimpri Chinchwad College of Engg. Savitribai \\ Phule Pune University, India
}

\author{
S.S. Sambare \\ Department of Computer Engg. \\ Pimpri Chinchwad College of Engg. Savitribai \\ Phule Pune University, India
}

\begin{abstract}
Wireless Sensor Network (WSN) consists of small, light weighted, low cost sensor nodes to monitor the presence of environmental and physical phenomena like wind speed, temperature etc. The nodes in the WSN have limited batterypower. Improving the lifetime is one objective of any wireless sensor network. There are different solutions to fulfill this objective. Clustering approach is one solution clustering algorithm is used to create the cluster of the nodes and select the cluster head to perform the data collection and transmission operation. LEACH (Low Energy Adaptive Clustering Hierarchy), Particle Swarm Optimization (PSO) and Artificial Bee Colony $(\mathrm{ABC})$ are examples of clustering algorithms. A sensor node requires more energy for transmission rather than sensing and processing the information. Hence, data compression is another solution used to transfer lesser bits to final location. In this paper, we proposed a model where we use both clustering algorithm and compression algorithm to increase the Life of Wireless Sensor Network. To form a cluster we use a PSO clustering algorithm and to compress the data into smaller bits we use a Huffman compression algorithm.
\end{abstract}

\section{Keywords}

clustering; WSN; compression; lifetime, energy.

\section{INTRODUCTION}

Wireless Sensor networks (WSN) are deployed in an environment to monitor and record the environmental changes. This network consists of ten to thousands of small nodes having sensing, computation and communication capabilities. The data collected by sensor nodes are transmitted node by node to the base station in multi-hop routing. The nodes in WSN are equipped with a limited battery power. Basically WSN is deployed in a harsh area such as deep sea, war region, etc. It is almost difficult and expensive to change or replace the battery regularly. Hence increasing the network lifetime is one of the objectives of WSN. One solution to fulfill this objective is to form clusters of nodes using clustering approach. In this approach, Cluster-Head $(\mathrm{CH})$ is selected from a group of sensor nodes and these $\mathrm{CHs}$ collects data from other nodes. Once data collection is completed $\mathrm{CH}$ send this data to the base station. LEACH, PSO [10] and ABC are clustering algorithms used in Wireless Sensor Network [2]. Network Lifetime can be enhanced a lot by using clustering approach. LEACH [13] is a cluster-based protocol that forms distributed clusters in which the nodes select themselves as cluster heads with some probability [1]. This algorithm runs periodically and every node has a chance to become a cluster head. LEACH operates itself into rounds, where each round consists of a setup phase and a steady phase. A setup phase consists of a cluster formation and steady phase consists of a data transmission. But, LEACH does not guarantee the desired number of cluster heads selected and cluster heads are not evenly positioned across the network [2] To find the optimal position of the sensor nodes, particle swarm optimization algorithms were used. This algorithm based on migration behaviors of bird flock in the process of searching food. This algorithm also works in rounds, where each round consists of setup phase and steady state phase [7]. During the setup phase all nodes send information about energy level to the base station and a node with sufficient energy is selected as a Cluster Head. Artificial Bee Colony algorithm is inspired by behavior of honey bees [2]. This algorithm consists of three bee groups as onlookers, scouts, employee bees and a food source [10]. Each employed bee is corresponding to one food source. An onlooker bee selects one of food sources and tries to improve the solution. A scout bee makes random search.

Sensor nodes senses, processes and transmits the data. But nodes consume more energy during transmission rather than monitoring and processing the data. Therefore, reducing the transmitted bits by using compression algorithm can be one solution for increasing Lifetime of WSN. Data compression is a technique used to minimize the quantity of bits transmitted. There are two types of compression algorithms as Lossy and Lossless Compression algorithm [9]. Lossy compression reduces a data by permanently discarding certain information, such as redundant information. Whereas, with lossless compression, every single bit of original data remains after the file is uncompressed. Run Length Encoding (RLE), Huffman Coding Technique, Lempel-Ziv-Welch (LZW) [8] is universal lossless data compression algorithms. These are Lossless compression algorithm that reduces bits by identifying and eliminating statistical redundancy. Lossless compression does not lose any information.

\section{RELATED WORK}

Lots of work has been done in Wireless Sensor Network to enhance the energy efficiency of sensor nodes. LEACH protocol has some limitations such as it can't cover large distances; $\mathrm{CH}$ selection is random and non-uniform distribution of $\mathrm{CH}$. To overcome this limitations paper [2] proposed a new modified LEACH protocol called as Time Based LEACH protocol. In TBLEACH algorithm $\mathrm{CH}$ is selected using random time interval and Nodes having the shortest time interval will become the cluster heads.

In paper $[3,11]$ author presents a new clustering approach of Wireless Sensor Network using Particle Swam Optimization. PSO clustering is used to minimize the intra-cluster distance and optimize the energy consumption of the network. The proposed method gives a higher network lifetime and gives more alive nodes as compared to LEACH.

In paper [4] author presents a novel energy efficient clustering mechanism, based on an artificial bee colony algorithm to increase the network lifetime. Artificial bee colony algorithm 
inspires from the intelligent foraging behavior of honey bee swarms. The clustering using $\mathrm{ABC}$ algorithm gives more performance than LEACH and PSO.

In paper [5] author proposed Huffman data compression algorithms. It is a lossless data compression algorithm helps to increase the lifetime of WSN by compressing data into smaller bits.

\section{PROPOSED WORK}

In the previous section we see there are different types of clustering and compression algorithms used to increase the lifetime of Wireless Sensor Network. These algorithms are successfully used in WSN to enhance the Lifetime of WSN. PSO algorithm give optimally distributed Cluster-Head and gives minimum distance between $\mathrm{CH}$ and non- $\mathrm{CH}$ nodes as compared to LEACH. It also gives more alive nodes than LEACH algorithm. Hence in our work we are using PSO clustering algorithm to select $\mathrm{CH}$ and form a cluster. In our proposed approach we use both PSO clustering algorithm and Huffman compression algorithm to obtain the best result. In our proposed approach first we create a cluster of nodes and select one Cluster-Head using a PSO clustering algorithm. After the formation of clusters, $\mathrm{CH}$ will collect the data from other nodes. This data will be compressed to smaller bits using Huffman compression algorithm before transmitting it to the base station.

\subsection{Proposed Algorithm}

Step1: Create Wireless Sensor Network with 1 base and $n$ nodes.

Step2: Select Cluster-Head and form a cluster of nodes using the PSO algorithm.

Step3: Process

1) Cluster of nodes and select one Cluster-Head using a clustering algorithm.

2) $\mathrm{CH}$ will collect data from other nodes.

3) This data will be compressed to smaller bits using compression algorithm before transmitting it to the base station.

Step4: Huffman data compression and data transmission is applied.

\subsection{Cluster Setup using PSO}

For $N$ number of sensor nodes, clusters of the nodes are formed as follows:

1. Initialize the network using $N$ sensor nodes to select the $\mathrm{CH}$ among eligible nodes.

2. Nodes having Maximum energy and Minimum distance is selected as Cluster Head.

3. For each node $n i, i=1,2, \ldots, N$. Calculate distance $d\left(n i, \mathrm{CH}_{k}\right)$ between node $n i$ and all cluster heads $\mathrm{CH}_{k}$. Where $\mathrm{CH}_{k}$ is a $\mathrm{CH}$ belong to cluster $C_{k}$ of particle $p$.

4. Assign node $n i$ to cluster head $\mathrm{CH}_{k}$ If

$$
d\left(n i, \underset{(1)}{\left.C H_{k}\right)}=\min \forall k=1,2, \ldots, k\left\{d\left(n i, C H_{k}\right)\right\}\right.
$$

5. Form the cluster of $\mathrm{CH}$ and their neighbor nodes.

After the formation of clusters and selection of $\mathrm{CH}$, nodes will send the data to the $\mathrm{CH}$. After collecting all data $\mathrm{CH}$ will transmit the data to base station.

\subsection{Huffman Compression algorithm for WSN.}

Huffman compression algorithm is lossless data compression algorithm. In this algorithm the data which occur more frequently is represented by a smaller set of bits and data that occur less frequently is represented by a large set of bits.
Huffman Algorithm reduces energy and a memory used in sensor node. The value measured by sensor, is entered into Analog-to-Digital converter (ADC) to transform into a binary representation $r_{i}$, on $\mathrm{R}$ bits, where $\mathrm{R}$ is the resolution of the ADC. The difference between actual measured value and the previous value is calculated as:

$$
d_{i}=r_{i}-r_{i-1}
$$

The value $d_{i}$ that has to be transmitted is composed of two sets of bits $s_{i} \mid a_{i}$ :

$s_{i}$ : the first set of bits codifies the number $n i$ of bits used to represent $d_{i}$. The value of $n_{i}$ is obtained from $d i$ as follows:

$$
\left.n_{i}=\log _{2}(|d i|)\right]
$$

But when $d_{i}=0, n_{0}$ is considered 0 .

$a_{i}$ : the second sequence of bits is the binary representation of $\mathrm{d}_{\mathrm{i}}$ and it can be generated in three ways:

1. When $d_{i}>0$, ai corresponds to the $n_{i}$ low-order bits of the two's complement representation of $d i$;

2. When $d_{i}<0$, ai corresponds to the $n_{i}$ low-order bits of the two's complement representation of $d_{i-1}$;

3. When $d_{i}=0, a_{i}$ has no representation and $s_{i}$ is coded as 00 .

The bit stream formed by the combination of $s_{i}$ and $a_{i}$ is transmitted to the next hop from the network.

\section{RESULTS AND DISCUSSION}

The proposed method is implemented and simulated using OMNET++ simulator. At the initial stage we create a network of 1 base station and 20 sensor nodes. The following figure gives the number of alive nodes for original PSO and compressed PSO.

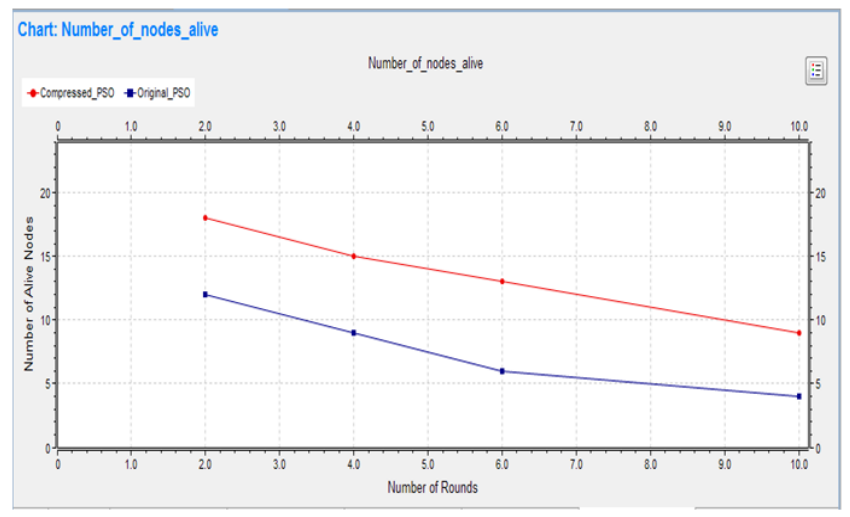

Fig. 1 Number of nodes alive for 20 Nodes

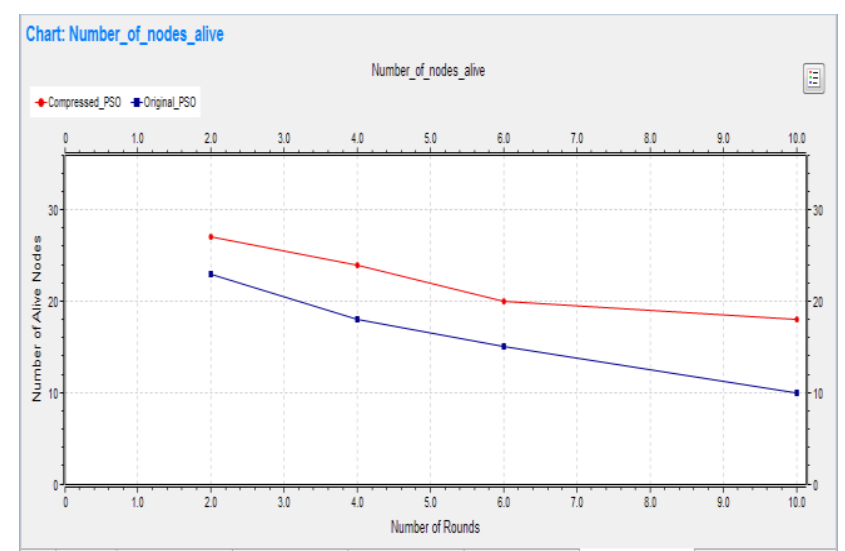

Fig. 2 Number of nodes alive for 30 Nodes 
Fig. 1and 2 shows the comparison of alive nodes for compressed PSO and original PSO. It shows that Compressed PSO gives more alive nodes than original PSO for 20 and 30 nodes respectively.

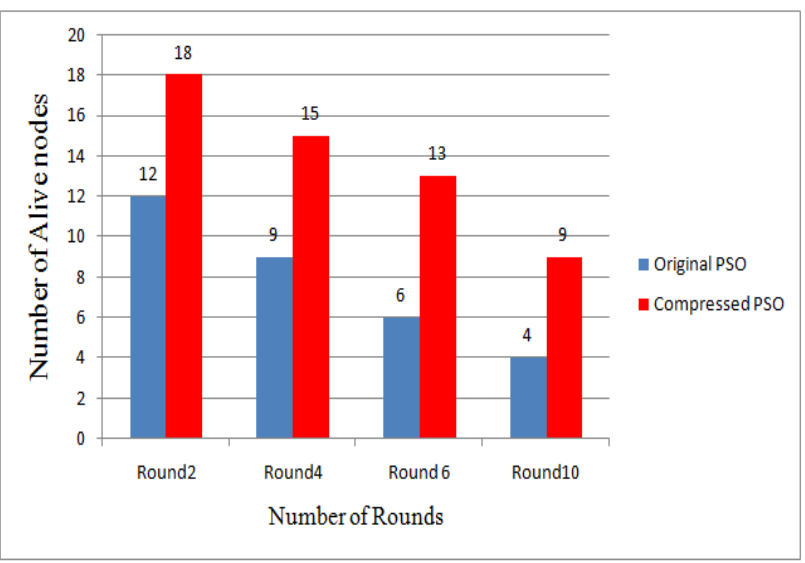

Fig. 3 Comparison between Original PSO and Compressed PSO for 20 nodes

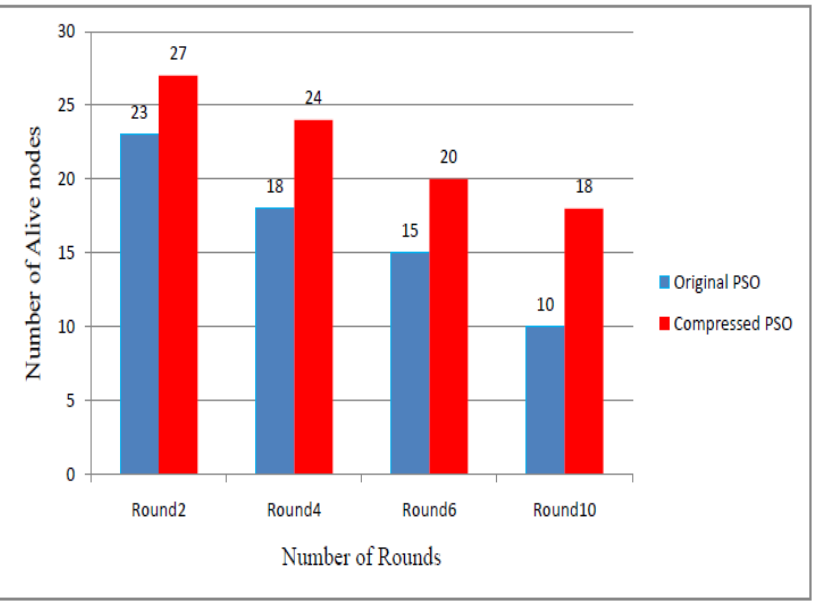

Fig. 4 Comparison between Original PSO and Compressed PSO for 30 nodes

Figure 3 and 4 gives the comparison between original PSO and compressed PSO for 20 nodes and 30 nodes for each round respectively. It shows that the after last round Compressed PSO gives more alive nodes than Original PSO.

\section{Performance of Proposed Model}

Increase in the percentage of alive nodes in Wireless Sensor Network after last round is given by:

$$
100 \times\left(1-\frac{N_{o}}{N_{c}}\right)
$$

Where, $N_{O}=$ Number of Alive nodes for Original PSO.

$N_{C}=$ Number of Alive nodes for Compressed PSO.

- For 20 nodes,

Increase in No. of alive nodes $=100\left(1-\frac{4}{9}\right)$ $=55 \%$

For 20 nodes, alive nodes increased by $55 \%$.
- For 30 nodes,

$$
\begin{gathered}
\text { Increase in No. of alive nodes }=100\left(1-\frac{10}{18}\right) \\
=44 \%
\end{gathered}
$$

For 30 nodes, alive nodes increased by $44 \%$.

\section{CONCLUSION AND FUTURE WORK}

From above result it is concluded that PSO with compression of data gives more alive nodes than PSO without compression of data. In our simulation, For 20 nodes it gives $55 \%$ of increase in number of alive nodes of Wireless Sensor Network and for 30 nodes it gives $44 \%$ of increase in number of alive nodes of Wireless Sensor Network. So, it is conclude that our proposed method can be used to increase the number of alive nodes and increases the Lifetime of Wireless Sensor Network.

In future work we can use the clustering using Artificial Bee Colony algorithm to increase the lifetime of wireless sensor network.

\section{REFERENCES}

[1] Nikhil Marriwala, Priyanka Rathee, "An Approach to Increase the Wireless Sensor Network Lifetime", 978-14673-4805-8/12/,pp. 495-499 IEEE 2012.

[2] Hu Junping, Jin Yuhui, Dou Liang, "A Time-based ClusterHead Selection Algorithm for LEACH", 978-1-4244-27031/08/\$25.00 @2008 IEEE.

[3] N. M. Abdul Latiff, C. C. Tsimenidis and B. S. Sharif, "Energy-Aware Clustering For Wireless Sensor Networks Using Particle Swarm Optimization", The 18th Annual IEEE International Symposium on Personal, Indoor and Mobile Radio Communications,, IEEE 2007.

[4] Dervis Karaboga, Selcuk Okdem andCelal Ozturk, "Cluster based wireless sensor network routing using artificial bee colony algorithm", published online: 24 April 2012 @ Springer Science+Business Media, LLC 2012.

[5] Francesco Marcelloni,, Massimo Vecchio, "A simple algorithm for Data compression in Wireless Sensor Network", IEEE COMMUNICATIONS LETTERS,10897798/08/@2008 IEEE.

[6] Eug`ene Pamba Capo-Chichi, Herv'e Guyennet andJeanMichel Friedt, "K-RLE : A new Data Compression Algorithm forWireless Sensor Network", Third International Conference on Sensor Technologies and Applications, pp. 502-507, IEEE 2009.

[7] Nikitha Kukunuru, Babu Rao Thella, Rajya Lakshmi Davuluri, "Sensor Deployment Using Particle Swarm Optimization", International Journal of Engineering Science and Technology Vol. 2(10), 2010, 5395-5401.

[8] Zhou Yan-li, Fan Xiao-ping, Liu Shao-qiang and Xiong Zhe-yuan, "Improved LZW Algorithm of Lossless Data Compression for WSN", 978-1-4244-5540-9/10, pp. 523527 @ IEEE 2010.

[9] Selcuk Okdem and Dervis Karaboga, "Routing in Wireless Sensor Networks Using an Ant Colony Optimization (ACO) Router Chip", Sensors 2009, 9, 909-921; doi:10.3390/s90200909, ISSN 1424-8220.

[10] Stefania Monica and Gianluigi Ferrari, "Particle Swarm Optimization for Auto-localization of Nodes in Wireless Sensor Networks" Springer-Verlag Berlin Heidelberg 2013 ICANNGA 2013, pp. 456-465. 
[11] Valeria Loscrí, Enrico Natalizio and Francesca Guerriero, "Particle Swarm Optimization Schemes Based on Consensus for Wireless Sensor Networks", MSWiM'12, October 21-25, 2012, Paphos, Cyprus. Copyright 2012 ACM 978-1-4503-1628-6/12/10.

[12] FabianCastaño, AndréRossi, MarcSevaux, NubiaVelasco, "A column generation approach to extend lifetime in wireless sensor networks with coverage and connectivity constraints"2013@Elsevier.

[13] Satyajeet R. Shinge, S. S. Sambare, " Survey of different Clustering Algorithms used to Increase the Lifetime of Wireless Sensor Networks", International Journal of Computer Application(IJCA), Vol. 108, December 2014, pp. 15-18. 